Commun. Korean Math. Soc. 27 (2012), No. 1, pp. 1-6

http://dx.doi.org/10.4134/CKMS.2012.27.1.001

\title{
ANSWERS TO LEE AND PARK'S QUESTIONS
}

\author{
Min Su Kang
}

Abstract. In [K. J. Lee and C. H. Park, Some questions on fuzzifications of ideals in subtraction algebras, Commun. Korean Math. Soc. 22 (2007), no. 3, 359-363], Lee and Park posed three questions. In this paper, the affirmative answers to their questions are provided, and characterizations of fuzzy ideals are investigated.

\section{Introduction}

B. M. Schein [8] considered systems of the form $(\Phi ; \circ, \backslash)$, where $\Phi$ is a set of functions closed under the composition "०" of functions (and hence $(\Phi ; \circ)$ is a function semigroup) and the set theoretic subtraction "\" (and hence $(\Phi ; \backslash)$ is a subtraction algebra in the sense of [1]). He proved that every subtraction semigroup is isomorphic to a difference semigroup of invertible functions. $B$. Zelinka [9] discussed a problem proposed by B. M. Schein concerning the structure of multiplication in a subtraction semigroup. He solved the problem for subtraction algebras of a special type, called the atomic subtraction algebras. Y. B. Jun et al. [3] introduced the notion of ideals in subtraction algebras and discussed characterization of ideals. In [2], Y. B. Jun and H. S. Kim established the ideal generated by a set, and discussed related results. Jun et al. discussed several notions and properties in subtraction algebras (see $[4,5,6]$ ). Lee and Park posed three questions in the paper [7]. The aim of this paper is to provide affirmative answers to these questions. We investigate several characterizations of fuzzy ideals in subtraction algebras.

\section{Preliminaries}

By a subtraction algebra we mean an algebra $(X ;-)$ with a single binary operation "-" that satisfies the following identities: for any $x, y, z \in X$,

(S1) $x-(y-x)=x$;

(S2) $x-(x-y)=y-(y-x)$;

(S3) $(x-y)-z=(x-z)-y$.

Received July 27, 2010.

2010 Mathematics Subject Classification. 03G25, 08A72.

Key words and phrases. subtraction algebra, ideal, fuzzy ideal. 
The last identity permits us to omit parentheses in expressions of the form $(x-y)-z$. The subtraction determines an order relation on $X: a \leq b \Leftrightarrow$ $a-b=0$, where $0=a-a$ is an element that does not depend on the choice of $a \in X$. The ordered set $(X ; \leq)$ is a semi-Boolean algebra in the sense of [1], that is, it is a meet semilattice with zero 0 in which every interval $[0, a]$ is a Boolean algebra with respect to the induced order. Here $a \wedge b=a-(a-b)$; the complement of an element $b \in[0, a]$ is $a-b$; and if $b, c \in[0, a]$, then

$$
\begin{aligned}
b \vee c & =\left(b^{\prime} \wedge c^{\prime}\right)^{\prime}=a-((a-b) \wedge(a-c)) \\
& =a-((a-b)-((a-b)-(a-c))) .
\end{aligned}
$$

In a subtraction algebra, the following are true (see [3]):
(a1) $(x-y)-y=x-y$.
(a2) $x-0=x$ and $0-x=0$.
(a3) $(x-y)-x=0$.
(a4) $x-(x-y) \leq y$.
(a5) $(x-y)-(y-x)=x-y$.
(a6) $x-(x-(x-y))=x-y$.
(a7) $(x-y)-(z-y) \leq x-z$.
(a8) $x \leq y$ if and only if $x=y-w$ for some $w \in X$.
(a9) $x \leq y$ implies $x-z \leq y-z$ and $z-y \leq z-x$ for all $z \in X$.
(a10) $x, y \leq z$ implies $x-y=x \wedge(z-y)$.
(a11) $(x \wedge y)-(x \wedge z) \leq x \wedge(y-z)$.

Definition 2.1 ([3]). A nonempty subset $A$ of a subtraction algebra $X$ is called an ideal of $X$, denoted by $A \triangleleft X$, if it satisfies:

(b1) $a-x \in A$ for all $a \in A$ and $x \in X$.

(b2) for all $a, b \in A$, whenever $a \vee b$ exists in $X$ then $a \vee b \in A$.

Proposition 2.2 ([3]). Let $X$ be a subtraction algebra and let $x, y \in X$. If $w \in X$ is an upper bound for $x$ and $y$, then the element

$$
x \vee y:=w-((w-y)-x)
$$

is a least upper bound for $x$ and $y$.

\section{Answers to Lee and Park's questions}

In what follows let $X$ be a subtraction algebra unless otherwise specified.

Definition 3.1 ([7]). A fuzzy set $\mu$ in $X$ is called a fuzzy ideal of $X$ if it satisfies:

(c1) $(\forall x, y \in X)(\mu(x-y) \geq \mu(x))$,

(c2) $(\forall x, y \in X)(\exists x \vee y \Rightarrow \mu(x \vee y) \geq \min \{\mu(x), \mu(y)\})$.

Proposition 3.2 ([7]). If a fuzzy set $\mu$ in $X$ satisfies

(c3) $(\forall x, a, b \in X)(\mu(x-((x-a)-b)) \geq \min \{\mu(a), \mu(b)\})$, then $\mu$ is a fuzzy ideal of $X$. 
Proposition $3.3([7])$. Let $\mu$ be a fuzzy set in $X$ such that

(c4) $(\forall x \in X)(\mu(0) \geq \mu(x))$,

(c5) $(\forall x, y, z \in X)(\mu(x-z) \geq \min \{\mu((x-y)-z), \mu(y)\})$.

Then we have

$$
(\forall a, x \in X)(x \leq a \Rightarrow \mu(x) \geq \mu(a)) .
$$

Theorem 3.4 ([7]). If $\mu$ is a fuzzy ideal of $X$, then

$$
(\forall \alpha \in[0,1])(U(\mu ; \alpha) \neq \emptyset \Rightarrow U(\mu ; \alpha) \triangleleft X),
$$

where $U(\mu ; \alpha):=\{x \in X \mid \mu(x) \geq \alpha\}$ which is called the level set of $\mu$.

Lee and Park posed the following questions.

Question. 1. Does any fuzzy ideal of a subtraction algebra satisfy condition (c3)?

2. Does any fuzzy ideal of a subtraction algebra satisfy condition (c5)?

3. Does the converse of Theorem 3.4 hold?

We provide affirmative answers to these questions. We first answer to the third question.

Theorem 3.5. Let $\mu$ be a fuzzy set in $X$ for which (3.2) is valid. Then $\mu$ is a fuzzy ideal of $X$.

Proof. If there are $a, b \in X$ such that $\mu(a-b)<\mu(a)$, then $\mu(a-b)<t_{a} \leq$ $\mu(a)$ for some $t_{a} \in(0,1]$. Thus $a \in U\left(\mu ; t_{a}\right)$, but $a-b \notin U\left(\mu ; t_{a}\right)$. This is a contradiction, and so $\mu(x-y) \geq \mu(x)$ for all $x, y \in X$. Assume that there exist $a, b \in X$ such that $a \vee b$ exists and $\mu(a \vee b)<\min \{\mu(a), \mu(b)\}$. Then $\mu(a \vee b)<$ $t_{0} \leq \min \{\mu(a), \mu(b)\}$ for some $t_{0} \in(0,1]$. It follows that $a, b \in U\left(\mu ; t_{0}\right)$ and $a \vee b \notin U\left(\mu ; t_{0}\right)$ which is a contradiction. Therefore (c2) is valid. Hence $\mu$ is a fuzzy ideal of $X$.

Now we answer to the second question.

Theorem 3.6. Every fuzzy ideal $\mu$ of $X$ satisfies the inequality (c5).

Proof. If we put $x=x-z$ in (a3), then $((x-z)-y)-(x-z)=0$, i.e., $(x-z)-y \leq$ $x-z$. If we put $x=y$ and $y=x-z$ in (a4), then $y-(y-(x-z)) \leq x-z$. Hence $x-z$ is an upper bound for $(x-z)-y$ and $y-(y-(x-z))$. It follows from Proposition 2.2, (S2), (S3) and (a2) that

$$
\begin{aligned}
& ((x-y)-z) \vee y \\
= & ((x-y)-z) \vee(y-0) \\
= & ((x-y)-z) \vee(y-(y-y)) \\
= & ((x-y)-z) \vee(y-(y-(x-z))) \\
= & ((x-z)-y) \vee(y-(y-(x-z))) \\
= & (x-z)-(((x-z)-(y-(y-(x-z))))-((x-z)-y))
\end{aligned}
$$




$$
\begin{aligned}
& =(x-z)-(((x-z)-((x-z)-y))-(y-(y-(x-z)))) \\
& =(x-z)-((y-(y-(x-z)))-(y-(y-(x-z)))) \\
& =(x-z)-0=x-z
\end{aligned}
$$

so from (c2) that

$$
\mu(x-z)=\mu(((x-y)-z) \vee y) \geq \min \{\mu((x-y)-z), \mu(y)\}
$$

for all $x, y \in X$.

We finally answer to the first question.

Theorem 3.7. Every fuzzy ideal $\mu$ of $X$ satisfies the inequality (c3).

Proof. Suppose that $\mu$ is a fuzzy ideal of $X$. Then the nonempty level set $U(\mu ; t)$ of $\mu$ is an ideal of $X$ for all $t \in(0,1]$. Let $\theta_{U(\mu ; t)}$ be a relation on $X$ defined by

$$
(\forall x, y \in X)\left((x, y) \in \theta_{U(\mu ; t)} \Leftrightarrow x-y \in U(\mu ; t), y-x \in U(\mu ; t)\right) .
$$

Then $\theta_{U(\mu ; t)}$ is a congruence relation on $X$. For any $a, b \in U(\mu ; t)$ and $x \in X$, we have $(x, x) \in \theta_{U(\mu ; t)},(a, 0) \in \theta_{U(\mu ; t)}$ and $(b, 0) \in \theta_{U(\mu ; t)}$. Hence

$$
(x-((x-a)-b), 0)=(x-((x-a)-b), x-((x-0)-0)) \in \theta_{U(\mu ; t)},
$$

and so $x-((x-a)-b) \in U(\mu ; t)$. It follows that

$$
\mu(x-((x-a)-b)) \geq \min \{\mu(a), \mu(b)\}
$$

for all $a, b, x \in X$ because if there exist $a_{0}, b_{0} \in X$ such that

$$
\mu\left(x-\left(\left(x-a_{0}\right)-b_{0}\right)\right)<\min \left\{\mu\left(a_{0}\right), \mu\left(b_{0}\right)\right\},
$$

then $\mu\left(x-\left(\left(x-a_{0}\right)-b_{0}\right)\right)<t_{0} \leq \min \left\{\mu\left(a_{0}\right), \mu\left(b_{0}\right)\right\}$ for some $t_{0} \in(0,1]$. Thus $a_{0} \in U\left(\mu ; t_{0}\right)$ and $b_{0} \in U\left(\mu ; t_{0}\right)$, but $x-\left(\left(x-a_{0}\right)-b_{0}\right) \notin U\left(\mu ; t_{0}\right)$. This is a contradiction.

\section{Characterizations of fuzzy ideals}

Using [7] and the previous section, we have the following characterizations of fuzzy ideals.

Theorem 4.1. For a fuzzy set $\mu$ in $X$, the following assertions are equivalent:

(1) $\mu$ is a fuzzy ideal of $X$.

(2) $\mu$ satisfies the condition (3.2).

(3) $\mu$ satisfies the condition (c3).

(4) $\mu$ satisfies the conditions (c4) and (c5).

Theorem 4.2. A fuzzy set $\mu$ in $X$ is a fuzzy ideal of $X$ if and only if it satisfies the condition (c4) and

$$
(\forall x, y \in X)(\mu(x) \geq \min \{\mu(x-y), \mu(y)\}) .
$$


Proof. Assume that $\mu$ is a fuzzy ideal of $X$. Then the condition (c4) is valid by [7, Proposition 3.4]. The condition (4.1) is by taking $z=0$ in (c5) and using (a2).

Conversely, let $\mu$ satisfy (c4) and (4.1). Note that

$$
(x-((x-a)-b))-b=(x-b)-((x-a)-b) \leq x-(x-a) \leq a,
$$

that is, $((x-((x-a)-b))-b)-a=0$ for all $x, a, b \in X$. It follows from (c4) and (4.1) that

$$
\begin{aligned}
& \mu(x-((x-a)-b)) \\
\geq & \min \{\mu((x-((x-a)-b))-b), \mu(b)\} \\
\geq & \min \{\min \{\mu(((x-((x-a)-b))-b)-a), \mu(a)\}, \mu(b)\} \\
= & \min \{\min \{\mu(0), \mu(a)\}, \mu(b)\} \\
= & \min \{\mu(a), \mu(b)\}
\end{aligned}
$$

for all $x, a, b \in X$. Therefore $\mu$ is a fuzzy ideal of $X$ by Proposition 3.2.

Theorem 4.3. For fixed elements $a, b \in X$, let $\mu_{a}^{b}$ be a fuzzy set in $X$ defined by

$$
\mu_{a}^{b}(x)= \begin{cases}t_{1} & \text { if }(x-a)-b=0, \\ t_{2} & \text { otherwise }\end{cases}
$$

for all $x \in X$ and $t_{1}, t_{2} \in(0,1]$ with $t_{1}>t_{2}$. Then $\mu_{a}^{b}$ is a fuzzy ideal of $X$.

Proof. Since $(0-a)-b=0$, we have $\mu_{a}^{b}(0)=t_{1} \geq \mu_{a}^{b}(x)$ for all $x \in X$. Let $x, y \in X$. If $(x-a)-b=0$, then $\mu_{a}^{b}(x)=t_{1} \geq \min \left\{\mu_{a}^{b}(x-y), \mu_{a}^{b}(y)\right\}$. Suppose that $(x-a)-b \neq 0$. If $(y-a)-b=0$ and $((x-y)-a)-b=0$, then

$$
\begin{aligned}
(x-a)-b & =((x-a)-b)-0 \\
& =((x-a)-b)-((y-a)-b) \\
& =((x-a)-(y-a))-b \\
& =((x-y)-a)-b=0,
\end{aligned}
$$

a contradiction. Hence $(y-a)-b \neq 0$ or $((x-y)-a)-b \neq 0$, and thus $\mu_{a}^{b}(y)=t_{2}$ or $\mu_{a}^{b}(x-y)=t_{2}$. It follows that

$$
\mu_{a}^{b}(x)=t_{2}=\min \left\{\mu_{a}^{b}(x-y), \mu_{a}^{b}(y)\right\}
$$

Hence, by Theorem $4.2, \mu_{a}^{b}$ is a fuzzy ideal of $X$.

Theorem 4.4. A fuzzy set $\mu$ is a fuzzy ideal of $X$ if and only if it satisfies:

$$
(\forall a, b, x \in X)(x-a \leq b \Longrightarrow \mu(x) \geq \min \{\mu(a), \mu(b)\}) .
$$


Proof. Assume that $\mu$ is a fuzzy ideal of $X$. Let $a, b, x \in X$ be such that $x-a \leq b$. Then $(x-a)-b=0$, and so

$$
\begin{aligned}
\mu(x) & \geq \min \{\mu(x-a), \mu(a)\} \\
& \geq \min \{\min \{\mu((x-a)-b), \mu(b)\}, \mu(a)\} \\
& =\min \{\min \{\mu(0), \mu(b)\}, \mu(a)\} \\
& =\min \{\mu(a), \mu(b)\}
\end{aligned}
$$

by $(4.1)$ and $(\mathrm{c} 4)$.

Conversely, let $\mu$ be satisfy the condition (4.2). Since $0-x \leq x$ for all $x \in X$, it follows from (4.2) that

$$
\mu(0) \geq \min \{\mu(x), \mu(x)\}=\mu(x)
$$

for all $x \in X$. Note that $x-(x-y) \leq y$ for all $x, y \in X$. Using (4.2), we have $\mu(x) \geq \min \{\mu(x-y), \mu(y)\}$ for all $x, y \in X$. Hence $\mu$ is a fuzzy ideal of $X$ by Theorem 4.2.

\section{References}

[1] J. C. Abbott, Sets, Lattices and Boolean Algebras, Allyn and Bacon, Boston 1969.

[2] Y. B. Jun and H. S. Kim, On ideals in subtraction algebras, Sci. Math. Jpn. 65 (2007), no. 1, 129-134.

[3] Y. B. Jun, H. S. Kim, and E. H. Roh, Ideal theory of subtraction algebras, Sci. Math. Jpn. 61 (2005), no. 3, 459-464, :e-2004, 397-402.

[4] Y. B. Jun, Y. H. Kim, and K. A. Oh, Subtraction algebras with additional conditions, Commun. Korean Math. Soc. 22 (2007), no. 1, 1-7.

[5] Y. B. Jun, C. H. Park, and E. H. Roh, Order systems, ideals and right fixed maps of subtraction algebras, Commun. Korean Math. Soc. 23 (2008), no. 1, 1-10.

[6] K. J. Lee, Y. B. Jun, and Y. H. Kim, Weak forms of subtraction algebras, Bull. Korean Math. Soc. 45 (2008), no. 3, 437-444.

[7] K. J. Lee and C. H. Park, Some questions on fuzzifications of ideals in subtraction algebras, Commun. Korean Math. Soc. 22 (2007), no. 3, 359-363.

[8] B. M. Schein, Difference semigroups, Comm. Algebra 20 (1992), no. 8, 2153-2169.

[9] B. Zelinka, Subtraction Semigroups, Math. Bohemica 120 (1995), no. 4, 445-447.

Department of Mathematics

HANYANG UNIVERSITY

SEOUl 133-791, Korea

E-mail address: sinchangmyun@hanmail.net 